\title{
ADAPTIVE TESTING FOR NONDESTRUCTIVE EVALUATION OF DEGRADED MATERIALS
}

\author{
Ivan Tomáš ${ }^{*}$ - Gábor Vértesy ${ }^{* *}$
}

\begin{abstract}
Method of Adaptive testing (AT) is described, as a way of optimization of experimental evaluation of degraded materials, which are measured nondestructively, indirectly via a convenient physical process. The general AT is illustrated by a wellexplored special case of magnetic adaptive testing (MAT) of strained steel. Degradation curves obtained from AT are regularly more sensitive and experimentally friendly than traditional descriptors of the used physical process itself. Any of the traditional descriptors of the physical process, being optimized for description of the process itself, is very probably not optimized for description of the investigated material degradation.
\end{abstract}

K e y w or d s: indirect evaluation, nondestructive evaluation, degraded materials, optimization

\section{INTRODUCTION}

Materials that have been exposed to changing temperature, to environmental harassment, to various chemical attacks, and to unplanned mechanical overloads, or construction materials, which simply have been in use for extended periods of time and experienced cyclic fatigue, gradually deteriorate and their properties change. Such service-induced degradation processes lead to increasing modifications in structure of the materials. To detect the degradation early enough, before the end of the material service-lifetime, requires sensitive non-destructive testing of the material during its industrial employment. The non-destructive testing of the industrially applied material pace of life must be regularly done by indirect methods of measurement. The optimum choice of the applied physical process and of its measured features, which are well linked to the inspected degradation of the inspected material, proves to be crucial for the success.

In the following text a systematic approach towards the problem of such optimization is described generally as Adaptive Testing (AT) of modified materials, and its concrete application is illustrated and discussed on the already well tested example of Magnetic Adaptive Testing (MAT) of mechanically stressed ferromagnetic steel samples.

\section{ADAPTIVE TESTING}

The way of Adaptive Testing of materials presents a very simple - but in the common practice rarely applied - attitude. Provided a physical process with an established link to the inspected material degradation, $\delta$, is selected, the question appears as to which ones from the available features of the applied physical process should be chosen as descriptors, $D_{i},(i=1,2, \ldots)$ for the most convenient indication of the actual $\delta$-value. In other words what are the best "degradation functions/curves, $D_{i}(\delta)$ ". And also, what best parameters/coordinates, $P_{j}$, $(j=1,2, \ldots)$ of the applied process to carry out the measurement at. In short, how to identify from all the possibly available degradation curves, $D_{i}\left(\delta, P_{j}\right)$, the optimum ones, ie such degradation curves, which are correlated to the inspected modification, $\delta$, of the material according to the best-wished criteria detailed earlier by the examiner. Evidently, the satisfactory criteria can be fulfilled not only by a solitary degradation curve, but frequently by several of them, often obtained from a single broad measurement. This shifts the AT method among the appreciated family of multi-parametric ways of experimental testing.

A number of optional physical processes are usually available for the given purpose, and once a satisfactory physical process is selected, it is always tempting to characterize the variation of $\delta$ of the inspected material by measurement of one of the well-established, traditionally acknowledged descriptors of the process. Alas however, any traditional feature of the used process - being probably very good for description of the process itself - does not have to be at all optimized as a descriptor of the actually investigated degradation, $\delta$, of the material. As an example from the field of ferromagnetism, the magnetic coercive force, $H_{\mathrm{C}}$, measured at room temperature, $T$, is often referred to as a reasonable indicator of plastic deformation strain and/or fatigue of ferromagnetic steel samples, see eg [1-3]. In the language used above, the measured degradation curve would be then $H_{\mathrm{C}}\left(\delta, h_{b}=H_{\mathrm{SAT}}\right.$, $B=0, T=20^{\circ} \mathrm{C}$ ), where $h_{b}$ is magnetic field amplitude of the applied hysteresis loop, $H_{\mathrm{SAT}}$ is the value of the applied field corresponding to the material magnetic saturation, and $B$ is magnetic induction of the sample. The

\footnotetext{
* Center of Advanced and Innovative Technologies, VSB-TUO, Ostrava, Czech Republic, tomas@fzu.cz; ${ }^{* *}$ Hungarian Academy of Sciences, Centre for Energy Research, Institute of Technical Physics and Materials Science, Budapest, Hungary
} 


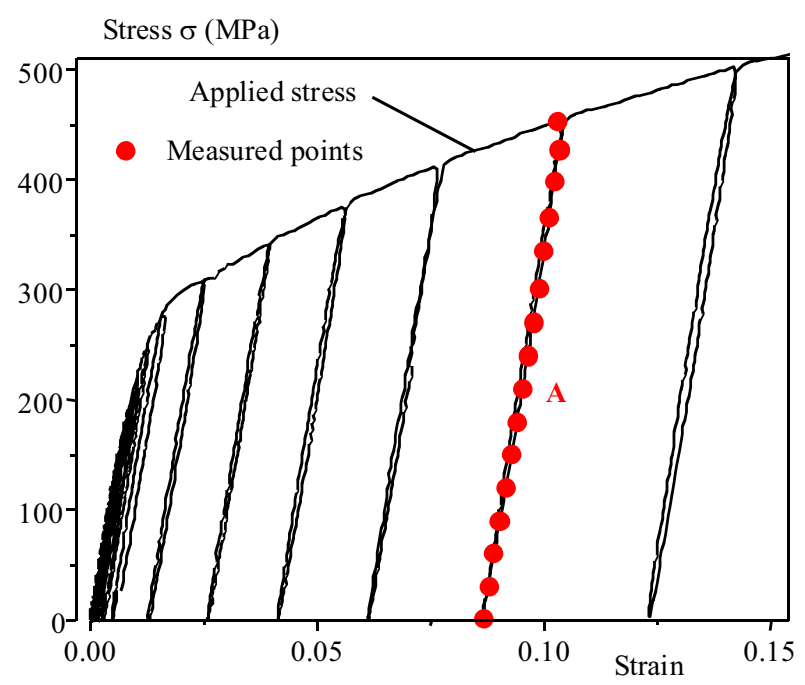

Fig. 1. The stress-strain diagram of the reference sample. The Abranch represents the elastic region of the sample (hardened by the earlier plastic deformation shown in the plot), which was presently used for the measurement and computation. The full circles show the stress-strain values, where the sample was measured magnetically.

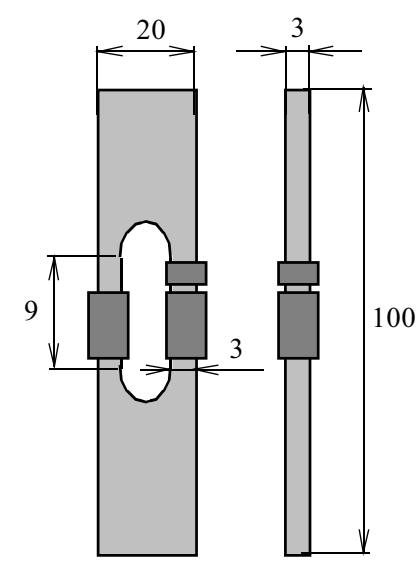

Fig. 2. The window-shaped steel sample. The top and bottom pads of the sample were clamped into the Instron holders. Two driving coils are positioned on each prismatic leg $(3 \times 3 \times 9 \mathrm{~mm})$, a pick-up coil sits on one leg. (This figure is not exactly scaled according to the correct dimensions in $\mathrm{mm}$.)

point is, however, that there exist a number of alternative degradation curves, which are more sensitive with respect to the plastic strain, $\delta$, and more measurement-friendly than $H_{\mathrm{C}}\left(\delta, h_{b}=H_{\mathrm{SAT}}, B=0, T=20^{\circ} \mathrm{C}\right)$, as shown $e g$ by application of Magnetic adaptive testing (MAT) in [4] or even in a broader scale in [5].

The simple recipe given by AT for finding the optimum degradation curve is the following:

1. Prepare a sequence of reference samples of the examined material with a step-by-step increased degradation, $\delta$.

2. Select a satisfactory physical process, known to be linked with $\delta$, and select which coordinates, $P_{j}$, to vary.

3. Carry out series of measurements on the sequence of the reference samples, and record the most suspected feature(s), $D_{i}$, of the process, while the selected coordinates, $P_{j}$, are varied in as broad frames as reasonably possible.

4. Arrange the measured data into indexed data-pools (eg to matrices), each corresponding to one relevant reference sample of the sequence, compute all available degradation curves $D_{i}\left(\delta, P_{j}\right)$, and pick up those, which best correspond to the wished criteria (eg experimental user-friendliness, sensitivity, robustness, special shape, minimum scatter, etc).

The optimum degradation curves are then used as the calibration curves during inspection of any unknown sample of the same material with the same expected type of degradation, $\delta$, as corresponds to the reference series of samples. The calibration curves are in this way optimally adapted to the inspected material, to its expected degradation, and to the desired experimental criteria. Any next tests of unknown samples are then carried out only at the optimum conditions as given by the values of the set of the coordinates $D_{i}\left(\delta, P_{1}, P_{2}, \ldots, P_{N}\right)$.

\section{EXAMPLE OF AT: MAGNETIC ADAPTIVE TESTING (MAT) OF STRESSED CONSTRUCTION STEEL}

In this Section an example of application of AT is explained, namely we describe an experimental search for the calibration curve/curves, best adapted to magnetic examination of a particular steel material, subjected to tensile stress within an elastic deformation range. Next testing of any unknown sample of the same kind of steel would be expected to indicate level of the currently applied mechanical tension through the value of the chosen magnetic feature of the material.

\subsection{Experiment}

The tested material was ferrite-pearlite low-alloyed steel $(0.23 \% \mathrm{C}, 0.91 \% \mathrm{Mn}, 0.31 \% \mathrm{Si}, 0.012 \% \mathrm{P}, 0.010 \% \mathrm{~S}$, $0.25 \% \mathrm{Cr}, \quad 0.39 \% \mathrm{Ni}, \quad 0.11 \% \mathrm{Mo}, \quad 0.01 \% \mathrm{Ti}, \quad 0.07 \% \mathrm{Cu})$ (the same as in [4]), which after normalized annealing was plastically and elastically deformed in a loading machine Instron 1362. The stress-strain diagram of the mechanical loading is shown in Fig. 1, where the long elastic branch (denoted here as A-branch) of the earlier plastically hardened sample was employed for measurement and for the search of the best calibration curves.

The series of reference samples for AT was represented in this case by a single sample of the inspected steel, which was consequently measured at the stress-points indicated by the 16 full circles in Fig. 1. As in the presented example the means of "degradation" of the material is the externally applied stress, the general AT-symbol of the degradation variable, $\delta$, is here substituted by the commonly used symbol for stress, $\sigma$. The procedure of magnetization of the material by an applied magnetic field was selected as the physical process for the indirect indication of the stress-level in the material. Strong links 


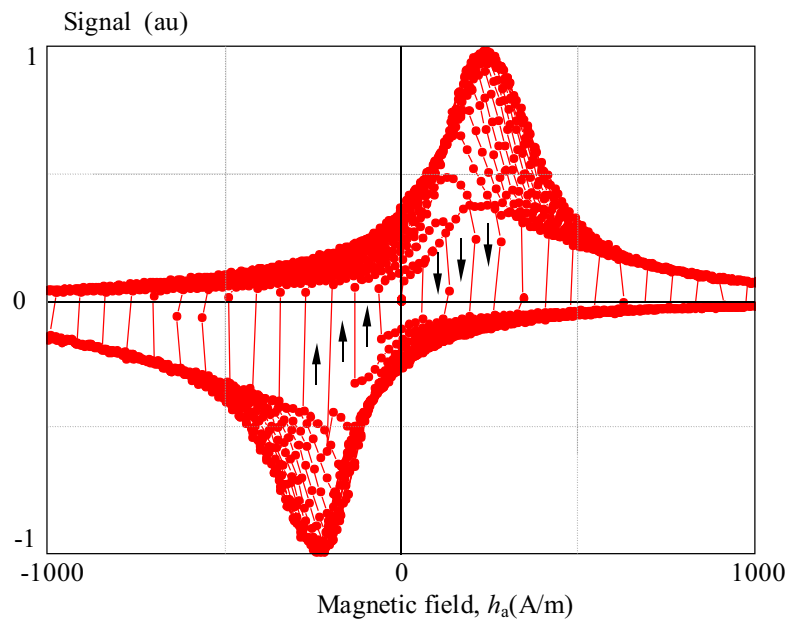

Fig. 3. The typical signal registered for one family of the triangular variations of the magnetizing field. The signal is proportional to a family of minor loops of differential permeability. The arrows show time development of the signal.

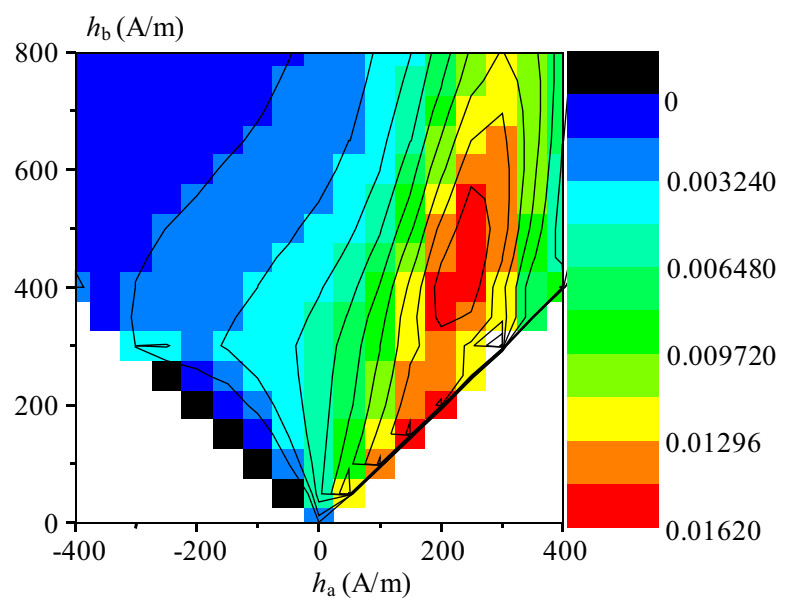

Fig. 4. Map of relative sensitivity of the $\mu$-degradation curves derived from the series of the permeability matrices, computed with steps $\Delta h_{a}=\Delta h_{b}=50 \mathrm{~A} / \mathrm{m}$. In the region depicted in the diagram the red color shows the most sensitive area. The most sensitive $\mu$ degradation curves for the investigated steel material under tensile stress in the elastic A-branch are obtained for the values of the AT-coordinates around positions $h_{a}=250 \mathrm{~A} / \mathrm{m}, h_{b}=400 \mathrm{~A} / \mathrm{m}$.

between mechanical stress, microstructure, and magnetic properties of ferromagnetic materials are well known and satisfactorily described, see eg [6]. The sample was prepared in a shape, which allowed it to be loaded by the tensile stress and at the same time to be magnetized in a closed magnetic circuit (in order to avoid complications connected with demagnetization of the magnetic open circuit configurations). Shape of the sample is sketched in Fig. 2 .

Now, the sample was installed into the clamps of the Instron, and stressed by tension according to Fig. 1. The elastic A-branch is the only part of the stressing procedure described by Fig. 1, which was applied within this paper for the calibration curves search: the first magnetic measurement was done at stress $\sigma=0 \mathrm{MPa}$, the second at stress $\sigma=30 \mathrm{MPa}$, etc up to $\sigma=450 \mathrm{MPa}$.
The magnetic measurement at each stress-point was started by a thorough demagnetization of the sample, and then it proceeded by magnetizing the sample "around the sample window" by the driving coils and by recording the voltage signal induced in the pick-up coil. The shape of the magnetizing field corresponded to triangular sweeps of the field, $h_{a}$, with step-by-step increasing amplitude, $h_{b}$, from 0 up to about $1500 \mathrm{~A} / \mathrm{m}$, which was close to $H_{\mathrm{SAT}}$ for the sample. Typical shape of the picked-up voltage signal is presented in Fig. 3. Detailed description of a similar measurement can be found in [4] and of many others in [5].

The sweeping magnetic field, $h_{a}$, and its amplitudes, $h_{b}$, in each respective minor loop were chosen as the AT coordinates of the measurement. The recorded AT feature of the magnetization process was the induced signal, which - under the mentioned experimental conditions (see also [4]) — was proportional to differential permeability, $\mu$, of the magnetic circuit in the tested material.

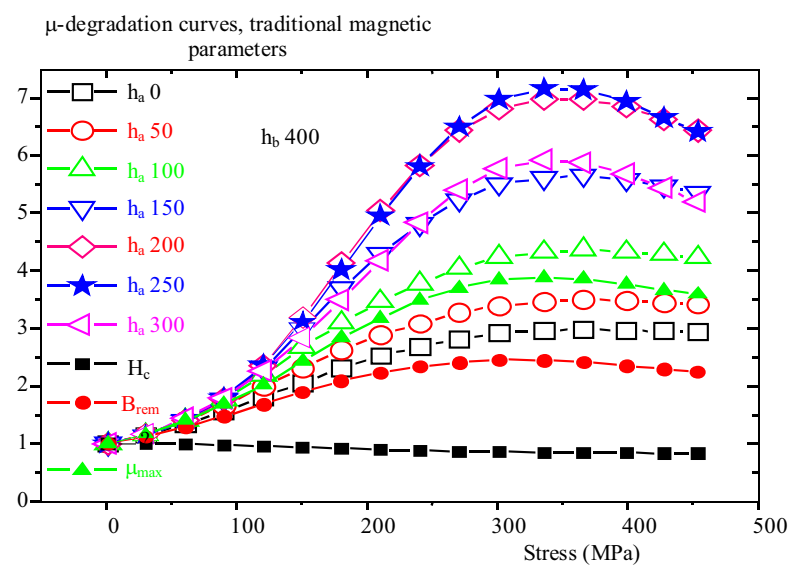

Fig. 5. Plot of the most sensitive $\mu$-degradation curve, $\mu\left(\sigma, h_{a}=\right.$ $250 \mathrm{~A} / \mathrm{m}, h_{b}=400 \mathrm{~A} / \mathrm{m}$ ), together with several other (less sensitive) $\mu$-degradation curves with the $h_{a}$-coordinates along the same minor loop amplitude, $h_{b}=400 \mathrm{~A} / \mathrm{m}$. Also the most sensitive traditional magnetic parameters $H_{\mathrm{C}}, B_{\mathrm{REM}}$ and $\mu_{\mathrm{MAX}}$ measured at the (almost) major hysteresis loop at $h_{b}=1200 \mathrm{~A} / \mathrm{m}$ are shown for comparison.

\subsection{Results}

Data of the type shown in Fig. 3 were recorded for each of the stress-points of the elastic A-branch in Fig. 1. The experimental raw data were smoothed, and interpolated into series of $\left(h_{a}, h_{b}\right)$ square matrices with preselected equal steps in the two directions (one matrix for each stress-point of the material). The stress-strain diagram (Fig. 1) and the series of the $\mu$-matrices completely described magnetic reflection of the variation of the sample elastically stressed along the A-branch. The series of matrices was then normalized by a reference matrix (matrix of the unstrained sample in A-branch), and all the mutually corresponding matrix elements in the whole series were arranged into $\mu$-degradation curves, $\mu\left(\sigma, h_{a}, h_{b}\right)$. Relative sensitivity of each $\mu$-degradation 


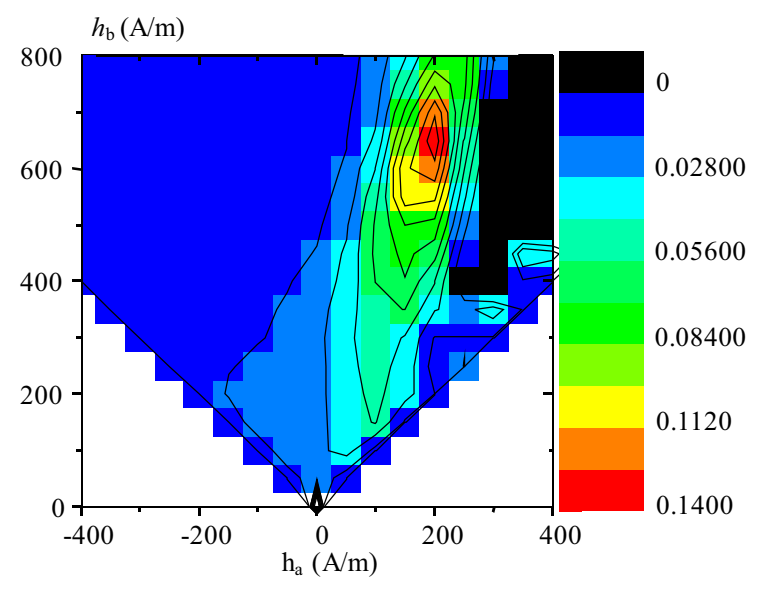

Fig. 6. Map of the relative sensitivity of the $\mu^{\prime}$-degradation curves derived from the series of the slope-of-the-permeability matrices, computed with steps $\Delta h_{a}=\Delta h_{b}=50 \mathrm{~A} / \mathrm{m}$. In the region depicted in the diagram the red color shows the most sensitive area. The most sensitive $\mu^{\prime}$-degradation curves for the investigated steel material under tensile stress in the elastic A-branch are obtained for the values of the AT-coordinates around positions $h_{a}=200 \mathrm{~A} / \mathrm{m}$, $h_{b}=650 \mathrm{~A} / \mathrm{m}$.

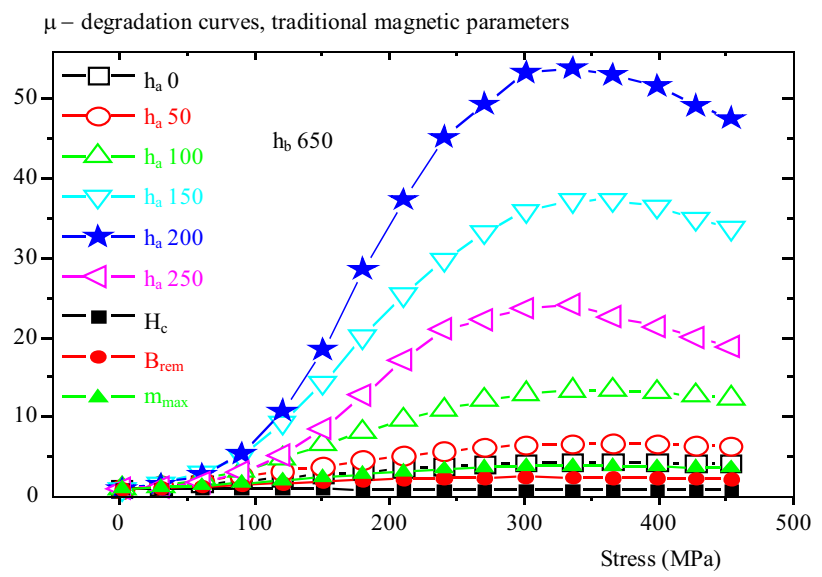

Fig. 7. Plot of the most sensitive $\mu^{\prime}$-degradation curve, $\mu^{\prime}\left(\sigma, h_{a}=\right.$ $200 \mathrm{~A} / \mathrm{m}, h_{b}=650 \mathrm{~A} / \mathrm{m}$ ), together with several other (less sensitive) $\mu^{\prime}$-degradation curves with the $h_{a}$-coordinates along the same minor loop amplitude, $h_{b}=650 \mathrm{~A} / \mathrm{m}$. Also the traditional magnetic parameters $H_{\mathrm{C}}, B_{\mathrm{REM}}$ and $\mu_{\mathrm{MAX}}$ are shown for comparison.

curve with respect to the applied stress, $\sigma$, was determined as slope of its linear regression and plotted in a sensitivity map shown in Fig. 4. The most sensitive $\mu$ degradation curve is plotted in Fig. 5 with some others, and together with the traditional magnetic parameters, namely coercive force, $H_{\mathrm{C}}$, remanent induction, $B_{\mathrm{REM}}$, and maximum permeability, $\mu_{\mathrm{MAX}}$. For details of the described data processing see again paper [4] and/or [5].

\section{DISCUSSION}

The elastic A-branch of Fig. 1 was chosen for illustration of the AT application. There was no other reason for choice of the material plastically hardened, as it had been that in the A-branch, than the fact, that the A-branch was long and it contained a large number of experimental points. Application of AT on other elastic branches of the same material brought qualitatively equivalent results.

The AT analysis presents a rather time-consuming measurement and evaluation of the recorded data. Such volume of measurement and of the following assessment would mean a heavy burden if this process would have to be used at every occasion of the material testing. In order to avoid misunderstanding, it is important to emphasize, that the AT analysis is carried out only once for the inspected type of the material and its degradation. The AT analysis, executed before the genuine material testing, establishes the optimum conditions for the ensuing tests ( $i e$ the optimum minor loop amplitude, $h_{b}$ ) and defines the optimum degradation curve(s) (ie the optimum value(s) of the sweeping field, $h_{a}$ ) as the calibration curve(s) to be applied further. The real testing of the material proceeds then fast, without need of any other AT analysis, just using its harvest.

Differential permeability, $\mu$, was selected in our example as the feature of the sample magnetization process, which was optimized by AT. As mentioned above and as described in more details in $[4,5]$, it is easy and advisable to broaden the investigation also by inspection of some "features of the feature", namely in the presented "magnetic" example of the integrated and differentiated values of $\mu$, ie the $B\left(h_{a}, h_{b}\right), \mu^{\prime}\left(h_{a}, h_{b}\right)$, etc. As an instructive example of this, Fig. 6 shows the sensitivity map for the $\mu^{\prime}$-degradation curves $\left(\mu^{\prime}=\mathrm{d} \mu\left(h_{a}, h_{b}\right) / \mathrm{d} h_{a}\right)$, while Fig. 7 gives an idea about shapes of some of the $\mu^{\prime}$-degradation curves around the local maximum of the sensitivity map, respectively.

As seen both from the sensitivity map in Fig. 6 and from the plots of the $\mu^{\prime}$-degradation curves neighboring the steepest one in Fig. 7, the sensitivity has a rather sharp peak at the maximum. Therefore, any incautious use of the single top sensitive $\mu^{\prime}$-degradation curve as a calibration curve for unknown samples testing can prove to be not reliable enough, unless the AT-coordinates $h_{a}$ and $h_{b}$ are determined with precision substantially better than the used experimental step of coordinates: better than the $50 \mathrm{~A} / \mathrm{m}$ for the discussed example of measurement. (Similar — even though less sharp — sensitivity peak is described also in Figs. 4, 5 for the $\mu$-degradation curves.)

This illustrates the fact, that beside the desired high sensitivity of a suitable degradation curve, also its stability/robustness with respect to fluctuation of the ATcoordinates values around the selected point is an important and required property. Stability of steepness of a degradation curve at an AT-coordinates point is determined by curvature of the sensitivity map surface at that point. Degradation curves defined at areas with high sensitivity and low curvature are generally preferred. Frequently it is desirable to find a compromise solution and/or to include other criteria, as for instance experimental friendliness of the selected calibration curve. A good example of such a compromise is shown in Fig. 8. 
$\mu-$ degradation curves, traditional magnetic parameters

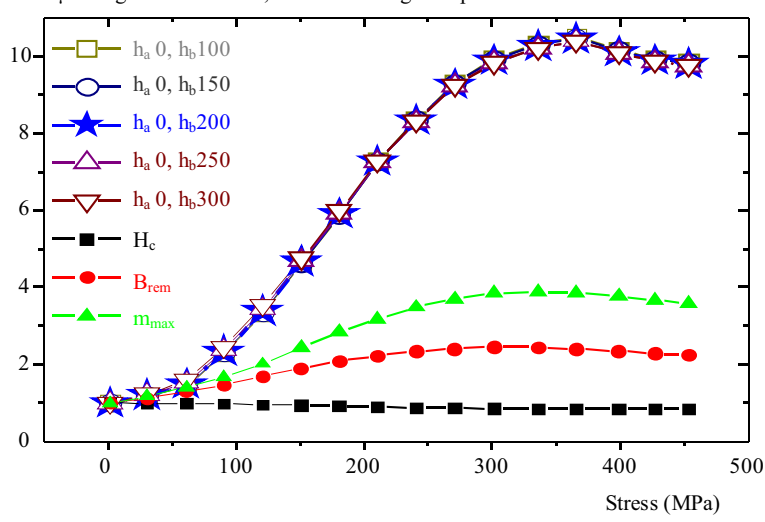

Fig. 8. Plot of a group of several equally sensitive $\mu^{\prime}$-degradation curves, $\mu^{\prime}\left(\sigma, h_{a}=0 \mathrm{~A} / \mathrm{m}, 100 \leq h_{b} \leq 300 \mathrm{~A} / \mathrm{m}\right)$ - see Figure 6 - which show high stability with respect to variation of the applied field amplitudes, and were measured at very low field amplitudes. The traditional magnetic parameters $H_{\mathrm{C}}, B_{\mathrm{REM}}$ and $\mu_{\mathrm{MAX}}$, measured at $h_{b}=1200 \mathrm{~A} / \mathrm{m}$, are shown for comparison of sensitivity.

Choice of the $\mu^{\prime}$-degradation curve at the AT-coordinates point $\left(h_{a}=0 \mathrm{~A} / \mathrm{m}, h_{b}=200 \mathrm{~A} / \mathrm{m}\right)$ yields the advantage of the high enough sensitivity (more than $1: 10$ change of magnitude at the stress variation from 0 up to about $370 \mathrm{MPa}$ ), very good stability with respect to the field amplitudes, $h_{b}$, and the selection of $h_{a}=0 \mathrm{~A} / \mathrm{m}$, which can be probably secured with even better precision than any non-zero field value. Besides, the required amplitude $h_{b}=200 \mathrm{~A} / \mathrm{m}$ of the magnetizing field is very userfriendly and makes it possible to be easily achieved even in experimental settings with magnetically open samples.

Beside this attitude of using degradation curves from the area of the sensitivity map with low curvature, another measuring strategy can be applied. Namely it is possible to utilize the advantage of multi-parametric output of the AT measurements. As seen from the family of the $\mu$-degradation curves in Fig. 5 and/or from a similar family of the $\mu^{\prime}$-degradation curves in Fig. 7, each of the families of those mutually different degradation curves is obtained from a single measurement (ie at a single minor loop amplitude $h_{b}$ ). However, each of the degradation curves is able to yield its corresponding AT-value to the same magnitude of the stress. In other words, the whole set of the AT-values from the set of the evaluated $h_{a^{-}}$ coordinates, ie the set of the degradation curves with different $h_{a}$ and the same $h_{b}$, can be used for determination of magnitude of the stress applied at an unknown sample. The stress, which is to be determined, can be thus established not from a single degradation/calibration curve, but from the whole set of them. This results in a multiparametric determination of the sought physical magnitude and as a consequence in a multiple confirmation of reliability of the result.

Not only this, however, is the advantage of such a measuring attitude: As can be seen both from Figs. 5 and 7 , both the $\mu$ - and the $\mu^{\prime}$ - top sensitivity degradation curves are not monotonous. This can make problems, provided the applied (and sought for) stress is larger than about $200 \mathrm{MPa}$ (for the here presented case). However, for example the $\mu$-degradation curves in Fig. 5 with coordinates $(0 \mathrm{~A} / \mathrm{m}, 400 \mathrm{~A} / \mathrm{m})$ and $(50 \mathrm{~A} / \mathrm{m}, 400 \mathrm{~A} / \mathrm{m})$ (and/or the $\mu^{\prime}$-degradation curve in Fig. 7 with coordinates $(0 \mathrm{~A} / \mathrm{m}, 650 \mathrm{~A} / \mathrm{m}))$ are monotonous and even though their sensitivity is not very high, it is high enough to solve the question of the stress having been in front of or behind the curve maximum. (Actually also $H_{\mathrm{C}}$ is monotonous and even though it is still less sensitive than the mentioned degradation curves, it can be used for the same purpose, if necessary.)

AT can be evidently successfully applied not only to magnetic testing of construction materials degraded by elastic and plastic deformation, by cyclic fatigue, and other industrial service influence (see eg [5]), but it is principally helpful in optimization of measurement of any characteristic of any varied material. It is not difficult to find a large number of possible applications. Beside the above described example of stress-responding ferromagnetic material measured within the broadest scale of its magnetization conditions, other materials can be tested under influence of other physical agents, like electrical field, etc. It is certainly advantageous if the physical process and its feature applied for the indirect AT measurement show hysteresis, as do for instance ferromagnetic and ferroelectric materials if acted upon by the corresponding fields with step-by-step varied amplitudes. The hysteresis yields different, unrepeated material states if the fields are repeated with different amplitudes, and this fact provides the broadest scale for the choice of the optimum AT coordinates.

However, nonexistence of hysteresis does not mean at all, that AT is not applicable, of course. Also physical qualities as eg temperature or pressure or others can be used as coordinates of an extra feature of a chosen physical process, and their multiple contemporary use can even make the AT method multidimensional.

Another example of sets of measurements - beside the so far tested MAT cases listed $e g$ in [5] — which were also well treated by the AT method, is for instance search for an optimum coordinate, $C_{n}$, of paramagnetic ions in $\mathrm{H}_{2} \mathrm{O} / \mathrm{D}_{2} \mathrm{O}$ solutions, used for very accurate measurement of magnetic field intensity by means of nuclear magnetic resonance. There, the optimum $C_{n}$-parameter was determined as a function of the measured field inhomogeneity, by regarding the highest value of the measurement accuracy, see [7].

As mentioned above, it happens in many cases of the AT measurements that not only a solitary one, but a whole group of different degradation curves looks to be quite satisfactory for the desired purpose. As such a family of satisfactory degradation curves is often obtained from a single measurement, it is very advantageous to use all of them as the "calibration curves" and thus to increase credibility of the tests by such a multi-parametric evaluation. 


\section{CONCLUSIONS}

This paper is dedicated to the optimization of indirect, nondestructive experimental measurement of variable material properties. In particular, it deals with the question how - from all available features of the physical process, which is employed for an indirect, nondestructive description of the investigated material variation - to determine that one, which is best adapted to the particular material under inspection, to the particular variation/degradation of the material, and to the particular demands declared by the examiner.

The introduced way of adaptive testing of materials suggests a procedure of collection of data on the selected physical process, whose parameters/coordinates are systematically modified in as broad ranges of values as to get the most complex picture of the behavior. Next analysis of the recorded data leads to a large family of degradation curves, the most satisfactory of which is/are then defined as the optimally adapted calibration curve (s) for next tests of unknown samples of the investigated material altered in the expected way.

As shown in the presented experimental example, an optimum selection among the degradation curves has to take into consideration not only their desired high sensitivity, but needs to demand also low experimental scatter of the curve-constituting measured values and low curvature of the sensitivity surface (referred to as stability/robustness of the calibration curve) around the selected AT-coordinates point. The easily achievable multiparametric data from even a single measurement strongly support reliability of the AT outcome. As the optimization of the AT measurements can proceed along many various examiner's demands, a number of alternative measuring and evaluation strategies can be applied for the top quality results.

The presented example obviously demonstrated that $\mathrm{AT}$, focusing on the explored concrete material and on the explored concrete degradation, introduces kind of a trade involving sensitivity, robustness, smoothness, shape, multi-parametricity, and experimental friendliness of the optimum calibration curves. This focus usually leads to excellent results of AT, which are substantially more advantageous and reliable for description of the explored material variations than the simple use of the traditional descriptors, which had been focused on the employed physical process itself.

\section{Acknowledgement}

The authors are indebted to V. Novák and O. Perevertov for measurement of the experimental data and to J. Kadlecová for her help with the computation. The assistance of Š. Barta who provided the samples, and of Z. Frait who kindly helped with his criticism to improve the text, is very much valued. The financial support of Hungarian Scientific Research Fund through project K 111662 is warmly appreciated (G.V.).

\section{REFERENCES}

[1] JOHnSON, M. J.-LO, C. C. H.-ZHU, B.-CAO, H.-JILES, D. C.: J. Nondestruct. Eval. 20 (2000), 11.

[2] JILES, D. C.: Magnetic Methods in Nondestructive Testing, in Encyclopedia of Materials Science and Technology (K. H. J. Buschow et al, eds.), Elsevier Press, Oxford, 2001, p. 6021.

[3] LO, C. C. H.-TANG, F.-JILES, D. C.-BINER, S. B. : J. Appl. Phys. 87 (2000), 6520.

[4] TOMÁŠ, I. : J.Mag.Mag.Mat. 268 (2004), 178.

[5] TOMÁŠ, I.-VÉRTESY, G. : Magnetic Adaptive Testing, Nondestructive Testing Methods and New Applications (Omar, M., ed.), 2012, (Rijeka: InTech) www.intechopen.com/ articles/show/title/magneticadaptive-testing.

[6] JILES, D. C.: J. Phys. D: Applied Physics 28 (1995), 1537.

[7] FRAit, Z.-FraitovÁ, D.: Czech. J. Phys. B27 (1977), 1292.

Received 28 July 2015

Ivan Tomáš (RNDr, PhD), born in Prague, Czech Republic, in 1943. Graduated from the Faculty of Mathematics and Physics at Charles University, Prague, in 1967, and received his PhD degree from the Czechoslovak Academy of Sciences in 1973. Presently he is with Institute of Physics, ASCR, Prague and at the Center of Advanced and Innovative Technologies, VSB-TUO, Ostrava. His main field is ferromagnetism of solids, with a strong interest in magnetic testing of variation and degradation of ferromagnetic industrial materials.

Gábor Vértesy (DSc, PhD), born in Budapest, Hungary, in 1951. Graduated from Eötvös Lornd University, Budapest, in 1974 and received his $\mathrm{PhD}$ degree from Hungarian Academy of Sciences in 1995. At present he is scientific advisor of Photonics Department of Hungarian Academy of Sciences, Centre for Energy Research, Institute for Technical Physics and Materials Science. His main field is study of ferromagnetic materials and development of novel nondestructive electromagnetic testing methods for characterization of structural industrial materials. 